\title{
Isomers of (L)-Diiodotyrosine - A DFT Treatise
}

\section{Lemi Türker}

Department of Chemistry, Middle East Technical University, Üniversiteler, Eskişehir Yolu No: 1, 06800 Çankaya/Ankara, Turkey; e-mail: 1turker@gmail.com; lturker@metu.edu.tr

\begin{abstract}
(L)-Diiodotyrosine isomers are considered within the realm of density functional theory at the level of B3LYP/6-311+G(d,p). Their zwitter ionic forms are considered as well. All the structures are electronically stable, have exothermic heat of formation and favorable Gibbs free energy of formation values. Within the limitations of the method the zwitter ionic forms are not different from the corresponding parent structures in the vacuum conditions and no hydrogen bonding seems to exist between the $\mathrm{NH}_{2}$ and $\mathrm{COOH}$ groups. Some structural, quantum chemical and spectral data have been collected and discussed.
\end{abstract}

\section{Introduction}

Thyroid hormones regulate gene expression, tissue differentiation and general development [1-3]. The thyroid gland produces two iodoamino acid hormones, 3,5,3'triiodothyronine (T3) and 3,5,3',5'-tetraiodothyronine (T4, Thyroxine). 3,5Diiodotyrosine, known as DIT, [1, 4] was discovered in thyroid in 1911 [5]. It has no biological activity relative to thyroxine (T4) [5].

Thyroglobuline is the precursor of $\mathrm{T} 3$ and $\mathrm{T} 4$. It is a huge, iodinated, glycosylated protein. About $70 \%$ of iodine in thyroglobuline exists in the inactive precursors, monoiodotyrosine (MIT) and diiodotyrosine (DIT) while $30 \%$ is in the iodothyronyl residues, T3 and T4. When iodine supplies are sufficient, T4:T3 ratio is about $7: 1[1,4]$.

Received: February 18, 2021; Accepted: March 24, 2021

Keywords and phrases: 3,5-diiodotyrosine, DIT, thyroid hormones, diiodotyrosine isomers, DFT.

Copyright (C) 2021 Lemi Türker. This is an open access article distributed under the Creative Commons Attribution License, which permits unrestricted use, distribution, and reproduction in any medium, provided the original work is properly cited. 

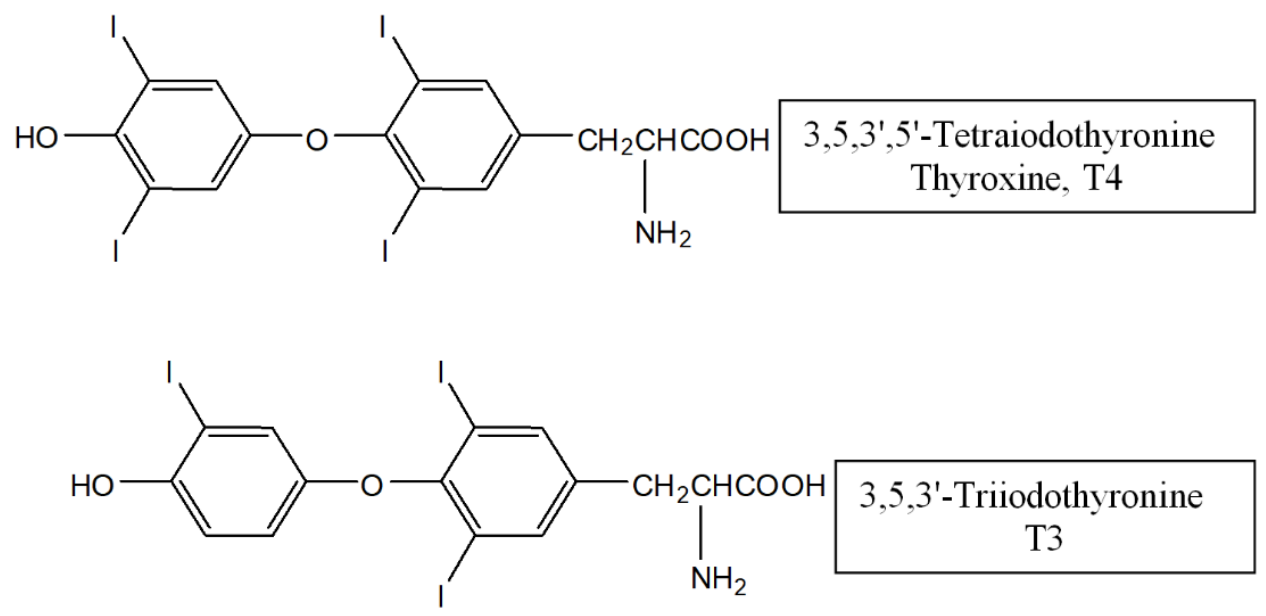

Diiodotyrosine (3,5-diiodotyrosine) is synthesized by directly iodinating tyrosine with iodine in the presence of sodium iodide in aqueous ethylamine, or in a mixture of acetic and hydrochloric acids with the addition of hydrogen peroxide $[6,7]$.

The formation of diiodotyrosine in iodinated human serum albumin was investigated by treating human serum albumin (HSA) with varying amounts of iodine under mild conditions [8].

Verification of antioxidative effect of 2,6-diiodotyrosine as homologue of thyroid hormone, T4 was done quantum chemically by Yanagida and coworkers [9].

To the best knowledge of the author very scarce of information exists in the literature about the isomers of diiodotyrosine except the 3,5-diiodotyronsine.

\section{Method of Calculation}

All the structures were subjected to MM2 initial geometry optimizations leading to energy minima followed by semi-empirical PM3 self-consistent fields molecular orbital (SCF MO) method $[10,11]$ at the restricted level [12]. Then, the structure optimizations have been achieved within the framework of Hartree-Fock (HF) and finally by using density functional theory (DFT) at the level of B3LYP/6-311+G(d,p) [13, 14]. The exchange term of B3LYP consists of hybrid Hartree-Fock and local spin density (LSD) exchange functions with Becke's gradient correlation to LSD exchange [15*38]. The correlation term of B3LYP consists of the Vosko, Wilk, Nusair (VWN3) local correlation 
functional [16] and Lee, Yang, Parr (LYP) correlation correction functional [17]. The normal mode analysis for each structure yielded no imaginary frequencies for the $3 \mathrm{~N}-6$ vibrational degrees of freedom, where $N$ is the number of atoms in the system which indicates that the structure of each molecule corresponds to at least a local minimum on the potential energy surface. Furthermore, all the bond lengths were thoroughly searched in order to find out whether any bond cleavage occurred or not during the geometry optimization process. All these computations were performed by using SPARTAN 06 [18].

\section{Results and Discussion}

Diiodotyrosine is one of the precursors of thyroid hormones. In the literature the name diiodotyrosine is mostly used instead of 3,5-diiodotyrosine isomer of diiodotyrosine family of structures. Figure 1 shows the diiodotyrosine isomers presently studied. It also shows the labels (A-D) standing for the isomers considered. Since in the structure of thyroid hormones, the tyrosine moiety has the S-configuration (L-form) the present study has been devoted to that form only.
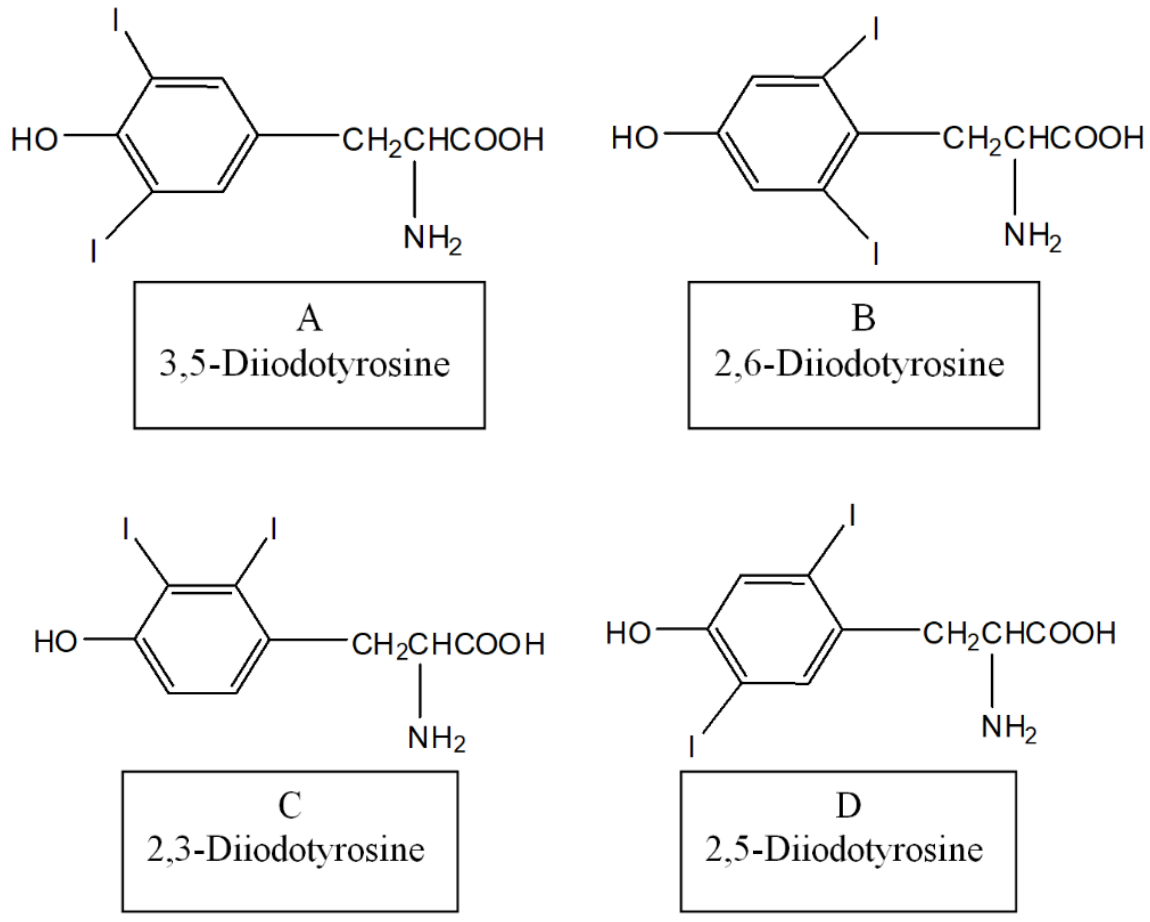

Figure 1. Isomers presently considered. 
Note that in structures A and B the iodine atoms are crossly conjugated with each other which is a property that restricts the possibility of extended conjugation in a $\pi$-skeleton.

Figure 2 shows the optimized structures of the isomers considered and their zwitter ionic forms which had been constructed from the parent structures, by transferring proton from carboxylic acid group to the amino group. The figure also shows the direction of the dipole moment vectors which originate from somewhere on the aromatic ring and points to the alkyl substituent. Note that in the optimized structures of the zwitter ionic forms one of the $\mathrm{N}-\mathrm{H}$ bond appears elongated relative to the others, implying its tendency to associate with the carboxylate oxygen atom. However, in the parent structures the carboxylic acid hydrogen faces to the $\mathrm{NH}_{2}$ group. In structure-A, the phenolic hydrogen faces to one of the iodine atoms rather than being equidistant to both of the iodines. A similar preference is observed in structure-C but not in structure-B. Note that in all the structures all the substituents (iodines, $\mathrm{OH}$ and $\mathrm{CH}_{2}$ ) are coplanar with the ring and no buttressing effect is observed [19].

As for the bond lengths, C-I bonds are about 2.13-2.16 $\AA$ in both of the parent compounds and their zwitter ionic forms, however in structures $\mathrm{zC}$ and $\mathrm{zD}$, the $\mathrm{C}-\mathrm{I}$ bond length for iodine atom at 2-position is somewhat longer than the other one.
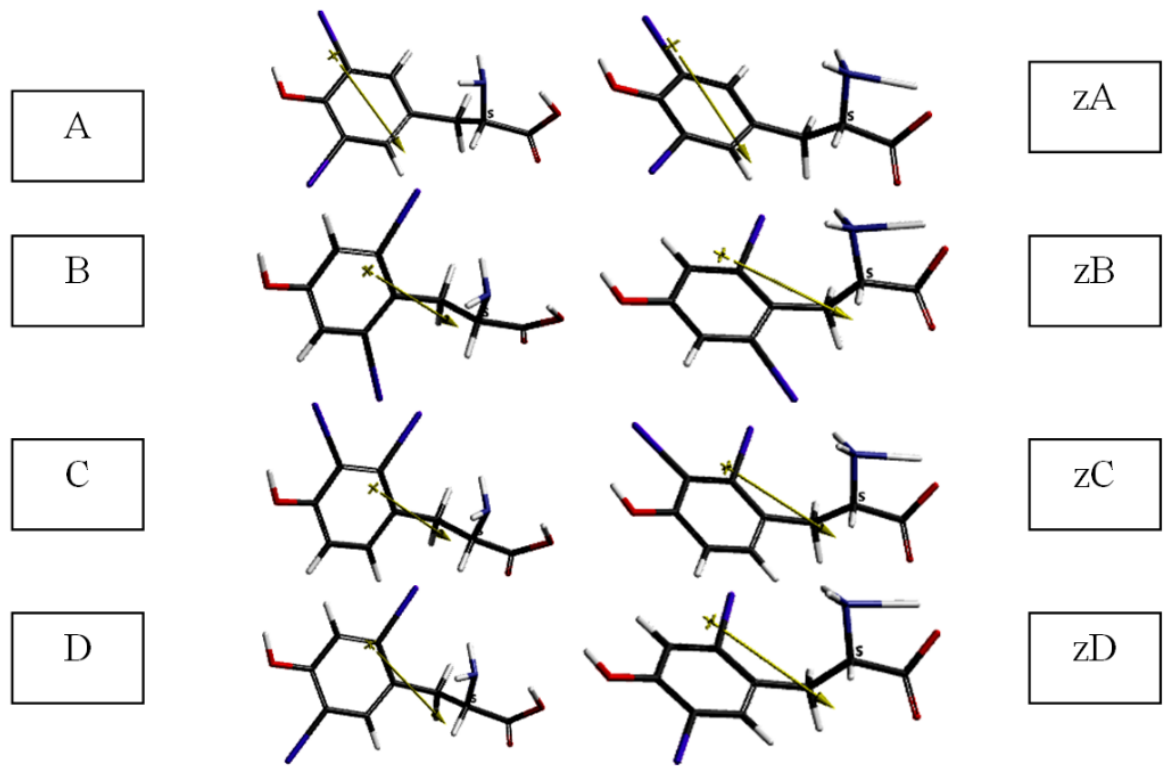

Figure 2. Optimized structures of the isomers and their zwitter ionic forms considered. 
Figure 3 displays the calculated IR spectra of the isomers presently considered. In all the spectrums the phenolic $\mathrm{O}-\mathrm{H}$ stretching precedes position of the $\mathrm{COO}-\mathrm{H}$ stretching and the last one is stronger than the phenolic one. The asymmetric N-H stretching lies in between the $\mathrm{O}-\mathrm{H}$ stretchings in all the cases and it is very weak.
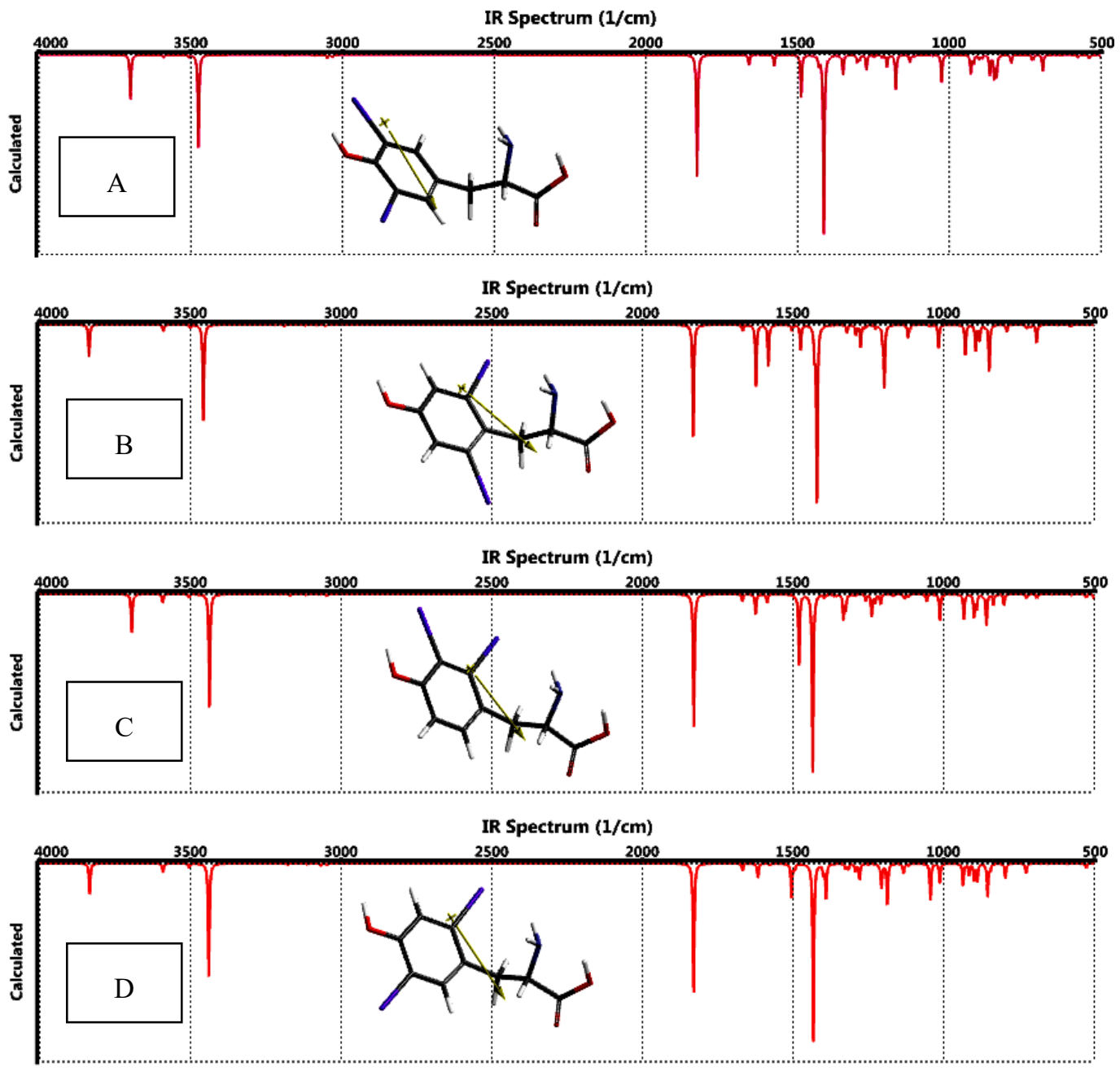

Figure 3. Calculated IR spectra of the isomers considered.

Table 1 lists some properties of the isomers which indicates that no appreciable differences exist between the parent structures and their zwitter ionic forms. There might be many reasons for it but most probable one could be that the proton transfer does not affect any conjugative path. Note that in the parent structures the hydrogen atom of $-\mathrm{OH}$ group of the carboxylic acid moiety faces to $\mathrm{NH}_{2}$ group already. 
Table 2 shows some thermodynamic properties of the isomers considered. According to the data in the table all of the isomers have exothermic heat of formation values and favorable Gibbs free energy of formation at the standard state.

Table 1. Some properties of the isomers considered.

\begin{tabular}{ccccccc}
\hline Isomer & $\begin{array}{c}\text { Dipole } \\
\text { moment }\end{array}$ & $\begin{array}{c}\text { Area } \\
\left(\AA^{\mathbf{2}}\right)\end{array}$ & $\begin{array}{c}\text { Volume } \\
\left(\AA^{\mathbf{3}}\right)\end{array}$ & Ovality & Log P & Polarizability \\
\hline $\mathbf{A}$ & 4.94 & 258.32 & 231.29 & 1.42 & 0.22 & 59.03 \\
$\mathbf{z A}$ & 4.88 & 258.33 & 231.28 & 1.42 & & 59.03 \\
$\mathbf{B}$ & 7.02 & 254.20 & 231.33 & 1.39 & 0.22 & 59.02 \\
$\mathbf{z B}$ & 7.02 & 254.05 & 231.32 & 1.39 & & 59.02 \\
$\mathbf{C}$ & 5.23 & 252.31 & 230.82 & 1.39 & 0.22 & 59.08 \\
$\mathbf{z C}$ & 5.23 & 252.21 & 230.82 & 1.39 & & 59.08 \\
$\mathbf{D}$ & 6.34 & 256.43 & 231.40 & 1.41 & 0.22 & 58.99 \\
$\mathbf{z D}$ & 6.34 & 256.50 & 231.40 & 1.41 & & 58.99 \\
\hline
\end{tabular}

Dipole moments in debye unit.

Table 2. Some thermodynamic properties of the isomers considered.

\begin{tabular}{cccc}
\hline Isomer & $\mathbf{H}^{\mathbf{0}}$ & $\mathbf{S}^{\mathbf{0}}\left(\mathbf{J} / \mathbf{m o l}^{\mathbf{0}}\right)$ & $\mathbf{G}^{\mathbf{o}}$ \\
\hline $\mathbf{A}$ & -37985715.16 & 485.86 & -37985860.09 \\
$\mathbf{z A}$ & -37985715.16 & 486.49 & -37985860.09 \\
$\mathbf{B}$ & -37985698.88 & 482.48 & -37985842.76 \\
$\mathbf{z B}$ & -37985698.88 & 482.41 & -37985842.50 \\
$\mathbf{C}$ & -37985697.83 & 477.81 & -37985840.14 \\
$\mathbf{z C}$ & -37985697.57 & 477.84 & -37985840.14 \\
$\mathbf{D}$ & -37985704.13 & 485.24 & -37985848.80 \\
$\mathbf{z D}$ & -37985704.13 & 485.33 & -37985848.80 \\
\hline
\end{tabular}

Energies in $\mathrm{kJ} / \mathrm{mol}$. In vacuum. 
Table 3 lists some energies of the isomers considered where $\mathrm{E}, \mathrm{ZPE}$ and $\mathrm{E}_{\mathrm{C}}$ stand for total electronic energy, zero point vibrational energy and the corrected total electronic energy, respectively. As indicated by data in the table, all the structures are electronically stable. The parent ones are slightly more stable than the corresponding zwitter ionic species except structure-A.

Figure 4 displays the electrostatic potential charges (ESP). Note that the ESP charges are obtained by the program based on a numerical method that generates charges that reproduce the electrostatic potential field from the entire wavefunction [18].

Table 3. Some energies of the isomers considered.

\begin{tabular}{cccc}
\hline Isomer & $\mathbf{E}$ & $\mathbf{Z P E}$ & $\mathbf{E}_{\mathbf{C}}$ \\
\hline $\mathbf{A}$ & -37985852.77 & 452.54 & -37985400.23 \\
$\mathbf{Z A}$ & -37985852.64 & 452.40 & -37985400.24 \\
$\mathbf{B}$ & -37985837.19 & 453.18 & -37985384.01 \\
$\mathbf{z B}$ & -37985837.20 & 453.29 & -37985383.91 \\
$\mathbf{C}$ & -37985836.74 & 454.21 & -37985382.53 \\
$\mathbf{Z C}$ & -37985836.77 & 454.30 & -37985382.47 \\
$\mathbf{D}$ & -37985842.55 & 453.18 & -37985389.37 \\
$\mathbf{Z D}$ & -37985842.56 & 453.21 & -37985389.35 \\
\hline
\end{tabular}

Energies in $\mathrm{kJ} / \mathrm{mol}$. In vacuum.

Figure 5 displays the electrostatic potential maps of the isomers considered. In the figure red/reddish and blue/ green regions stand for negative and positive potential fields, respectively.

Figure 6 shows the bond densities of the isomers considered. As seen in the figure any hydrogen bonding possibility in vacuum conditions is less likely between the $\mathrm{NH}_{2}$ and $\mathrm{COOH}$ groups because the proton transfer to form zwitter ion is actually results in charge separation. In the vacuum conditions the resultant anion and cation centers cannot be stabilized in contrast to the case of water. 

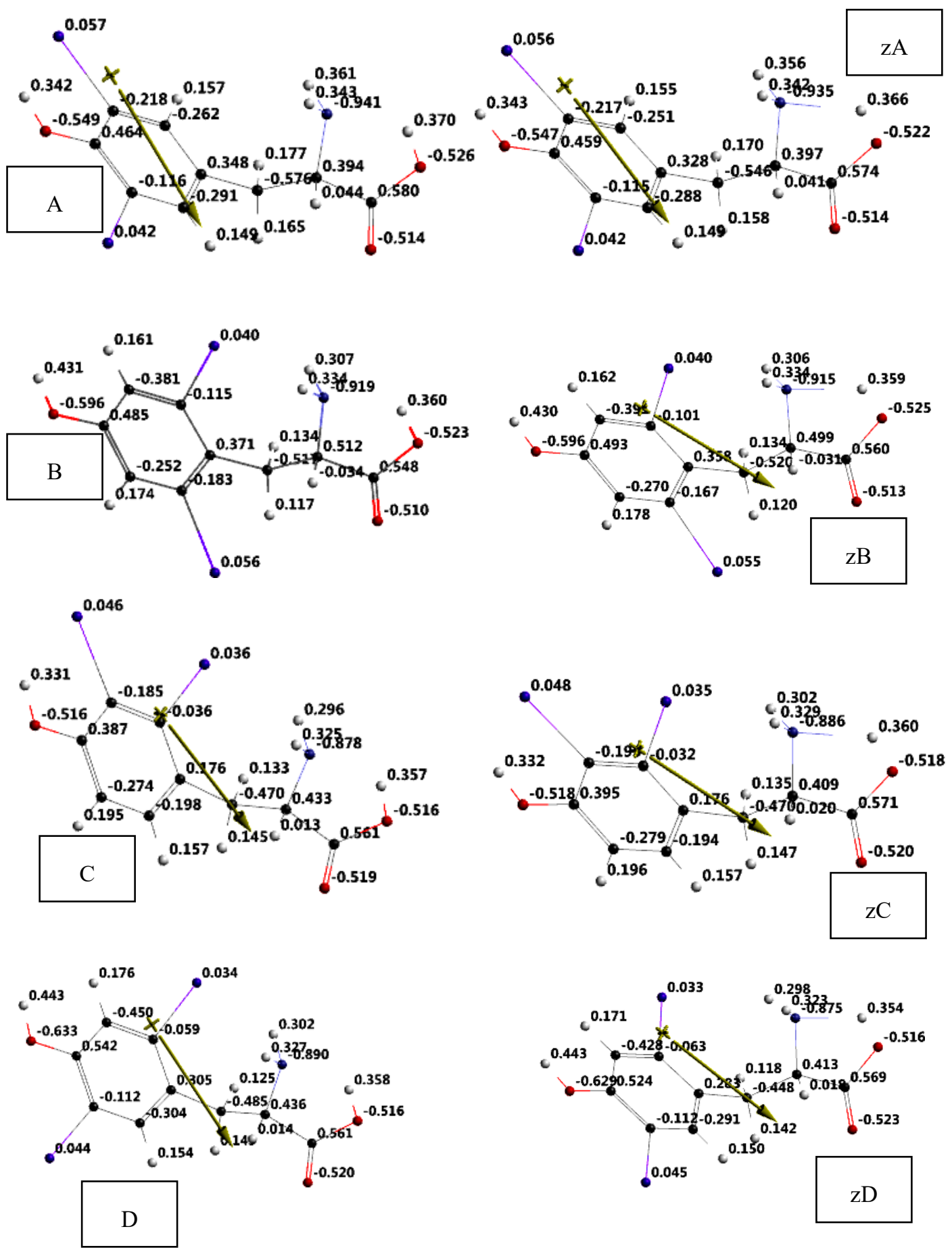

Figure 4. ESP charges on atoms of the structures considered. 

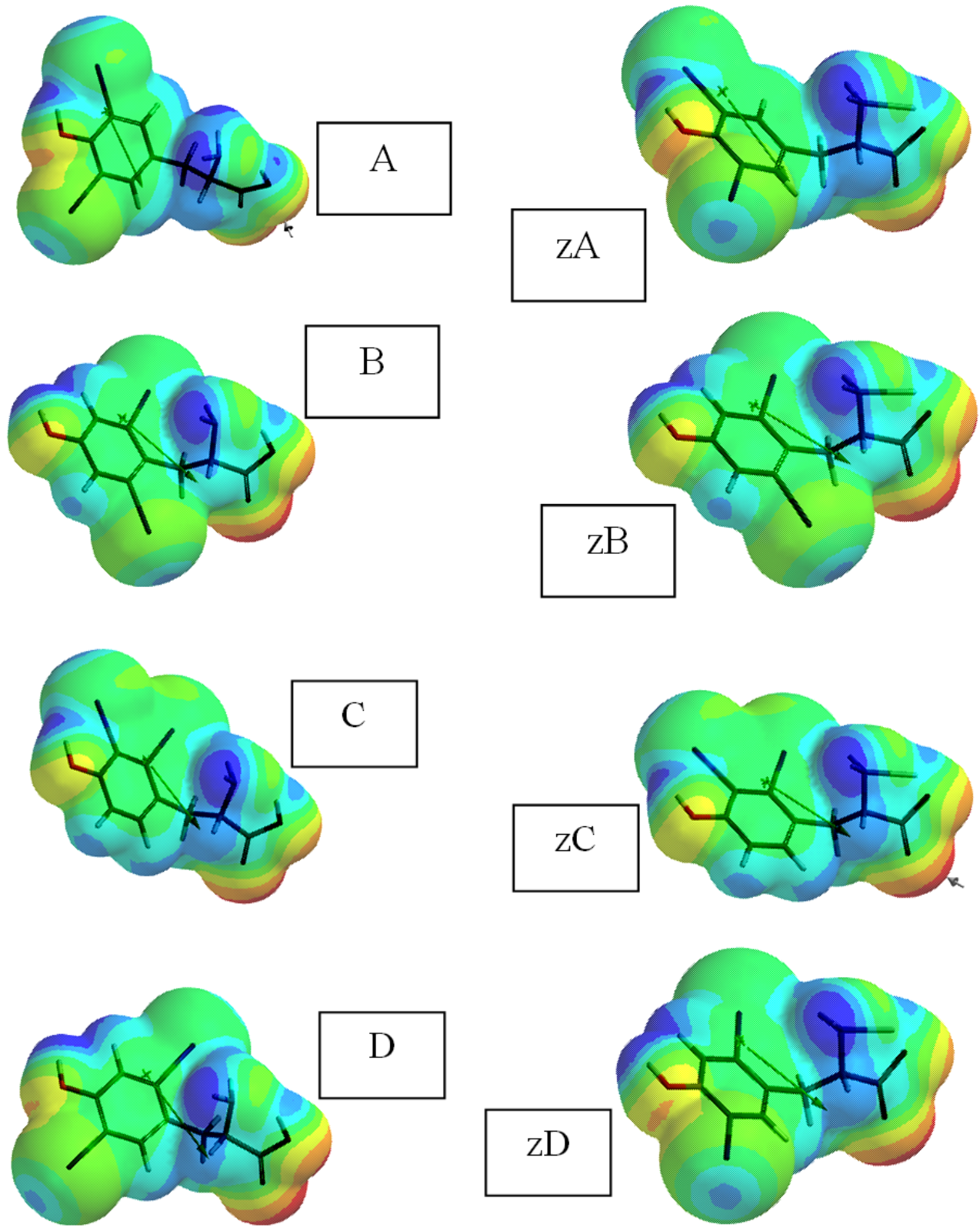

Figure 5. ESP maps of the structures considered.

Figure 7 depicts the molecular orbital energy spectra of the isomers. They differ from each other, especially the way of inner-lying molecular orbital energy levels. The frontier molecular orbital energies $\left(\varepsilon_{\text {Hомо }}\right.$ and $\left.\varepsilon_{\text {LUMO }}\right)$ are listed in Table 4 . As seen in the table the HOMO energies of the parents and the corresponding zwitter ionic structures are either 
equal or slightly different. Consequently, the $\Delta \varepsilon$ (interfrontier molecular orbital energy gap, $\Delta \varepsilon=\varepsilon_{\text {LUMO }}-\varepsilon_{\text {HOMO }}$ ) values for the parent and its zwitter ionic form differ slightly.
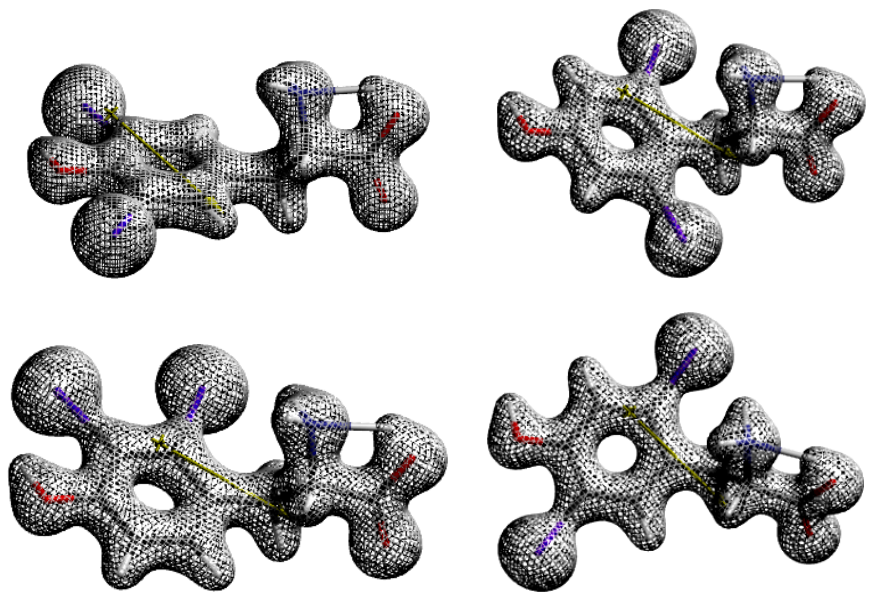

Figure 6. Bond densities of the isomers considered.
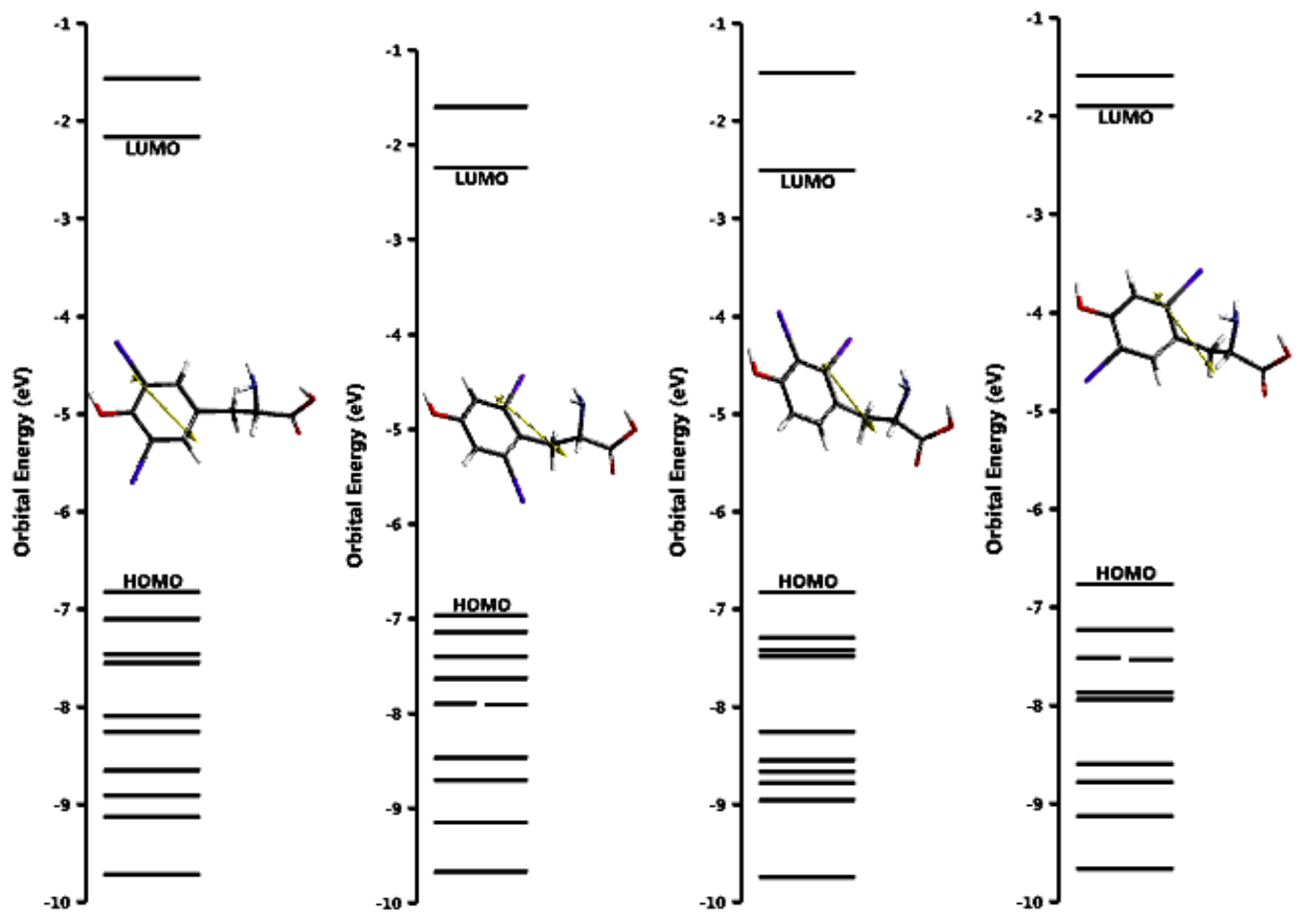

Figure 7. The orbital energy spectra of the isomers considered. 
Table 4. The HOMO, LUMO energies and $\Delta \varepsilon$ values of the isomers considered.

\begin{tabular}{cccc}
\hline Isomer & HOMO & LUMO & $\Delta \boldsymbol{\varepsilon}$ \\
\hline $\mathbf{A}$ & -658.20 & -208.74 & 449.46 \\
$\mathbf{Z A}$ & -658.60 & -208.67 & 449.93 \\
$\mathbf{B}$ & -671.84 & -215.92 & 455.92 \\
$\mathbf{z B}$ & -671.84 & -216.23 & 455.61 \\
$\mathbf{C}$ & -658.35 & -241.68 & 416.67 \\
$\mathbf{z C}$ & -658.21 & -241.76 & 416.45 \\
$\mathbf{D}$ & -652.47 & -182.94 & 469.53 \\
$\mathbf{z D}$ & -652.27 & -182.45 & 469.82 \\
\hline
\end{tabular}

Energies in $\mathrm{kJ} / \mathrm{mol}$. In vacuum.

The order of HOMO energies is $\mathrm{B}<\mathrm{C}<\mathrm{A}<\mathrm{D} ; \mathrm{zB}<\mathrm{zC}<\mathrm{zA}<\mathrm{zD}$ whereas the LUMO energies follow the order of $\mathrm{C}<\mathrm{B}<\mathrm{A}<\mathrm{D} ; \mathrm{zC}<\mathrm{zB}<\mathrm{zA}<\mathrm{zD}$. These orders should be dictated mainly by the position of the iodine atoms on the ring as well as their relative positions to each others. It is worth noting that iodine atom has $1 s^{2} 2 s^{2} 2 p^{6} 3 s^{2} 3 p^{6} 3 d^{10} 4 s^{2} 4 p^{6} 4 d^{10}$ electronic configuration in the ground state and its lone pair electrons are expected to be poorly conjugated with the ring due to orbital size mismatch of carbon and iodine atoms. Iodine also cannot exert effective inductive effect. The order of $\Delta \varepsilon$ values is $\mathrm{C}<\mathrm{A}<\mathrm{B}<\mathrm{D}$ for the parents as well as for their zwitter ionic forms.

Figure 8 shows the HOMO and LUMO patterns of the isomers. In the case of structure-A, only one of the iodines contribute to the HOMO. In the other cases, one of the iodines contributes less than the other ones. As for the LUMOs, the main contribution comes from iodines and the aromatic part of the structures. The carboxylic acid part does not contribute any. In structure-D, only one of the iodines contributes to the LUMO. 


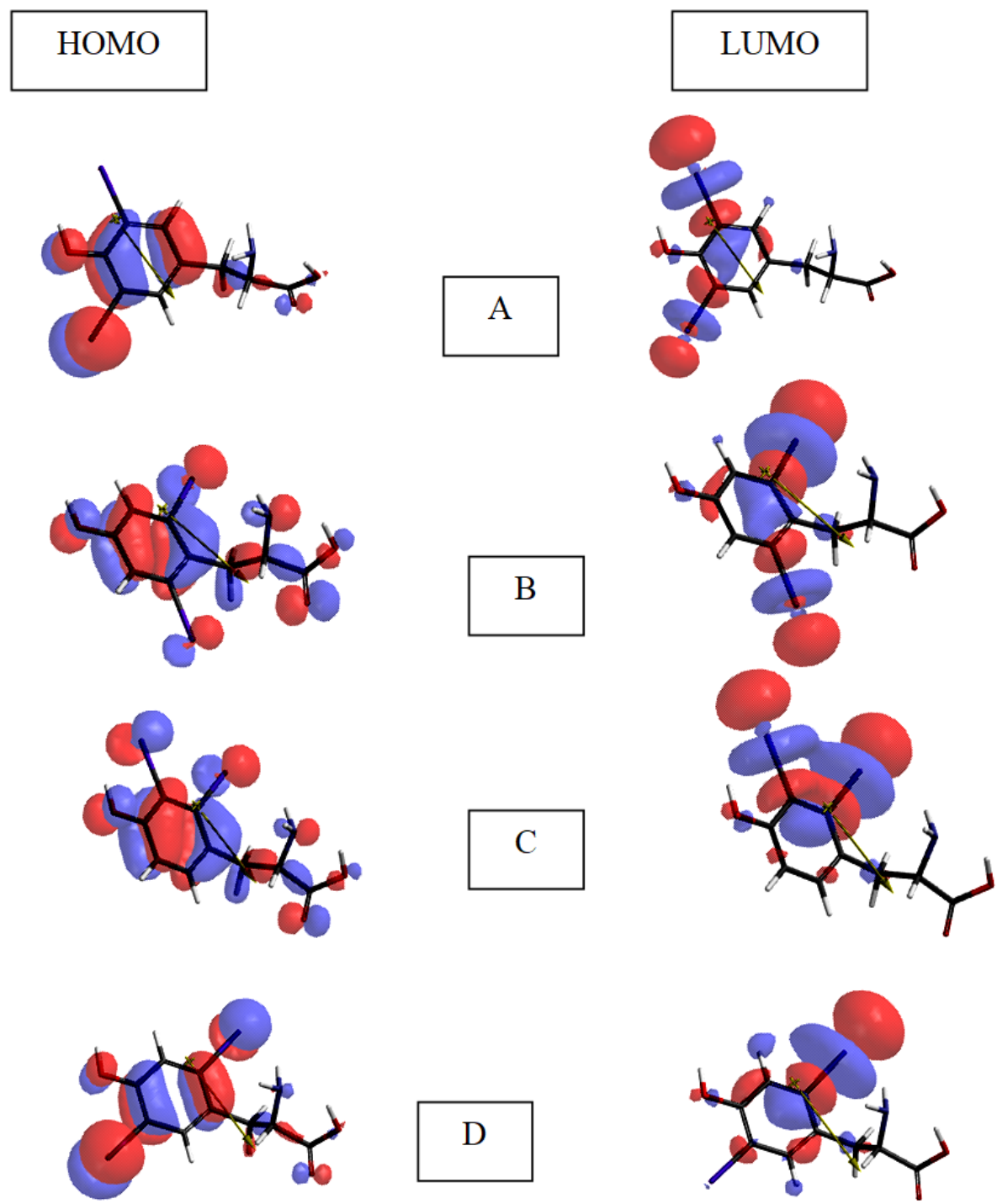

Figure 8. The HOMO and LUMO patterns of the structures considered.

Figure 9 shows the calculated time-dependent UV-VIS spectra of the isomers. As seen in the figure, going from structure-A to C, the slight bathochromic shift [19] occurs but the spectrum of structure-D is confined to UV region only. As expected from the order of $\Delta \boldsymbol{\varepsilon}$ values (see Table 4) which is $\mathrm{C}<\mathrm{A}<\mathrm{B}<\mathrm{D}$, the HOMO-LUMO electronic transition in the case of isomer-D is the most energy demanding whereas the least transition energy required in the case of isomer-C. The probable cause of the smallest $\Delta \varepsilon$ value of structure- $\mathrm{C}$ could be due to presence of the longest extended conjugation 
existing from iodine to iodine whereas it is the shortest path in structure-D. It is worth mentioning that interfrontier molecular orbital energy gap $(\Delta \varepsilon)$ decreases as the extended conjugation in a $\pi$-skeleton increases [20].
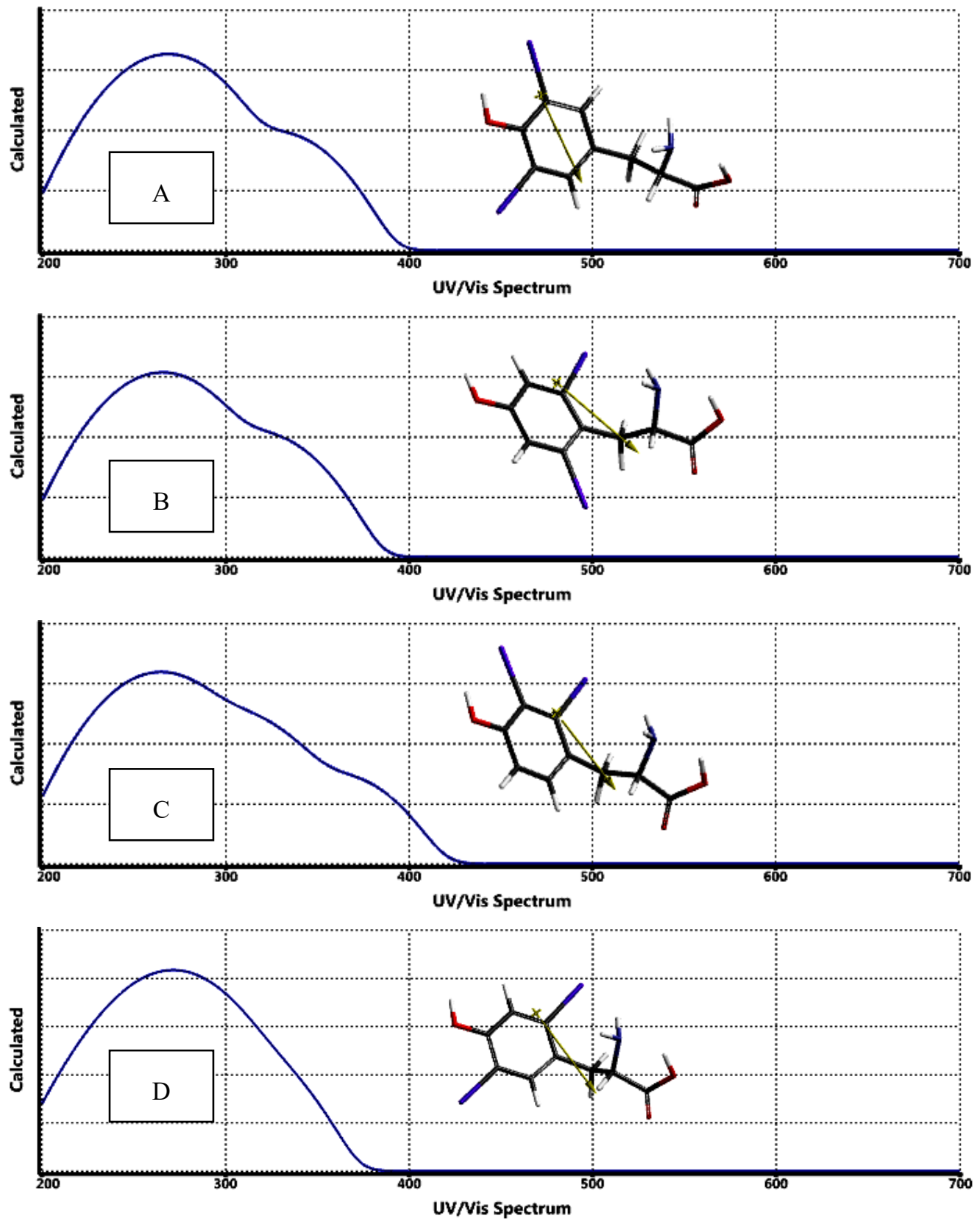

Figure 9. Calculated time-dependent UV-VIS spectra of the isomers considered. 


\section{Conclusion}

The present DFT treatise on (L)-diiodotyrosine isomers and their corresponding zwitter ionic forms has revealed that they are electronically stable and thermodynamically favorable within the limitations of the theory and the level of calculations. Although, the parent structures and their corresponding zwitter ionic forms initially had been constructed from different stand points, the optimized structures highly resemble each other. Namely, the zwitter ionic forms are optimized nearly to their parent structures. Probably, on the potential energy surface, the parent and its zwitter ionic form reside on nearly located energy valleys. Of course, in aqueous systems the zwitter ionic species which have charge separation could be stabilized due to solvolysis, thus a different picture may arise.

\section{References}

[1] D.K. Granner, Thyroid Hormones, in: Harper's Biochemistry, 23rd ed., Eds.: R.K. Murray, D.K. Granner, P.A. Mayers and V.W. Rodwell, California: Appleton and Lange, 1993.

[2] K. Venkatesh, L. Srikanth, B. Vengamma, C. Chandrasekhar, B. Chandra, M. Prasad and P.V.G.K. Sarma, In vitro transdifferentiation of human cultured CD34+ stem cells into oligodendrocyte precursors using thyroid hormones, Neuroscience Letters 588 (2015), 36-41. https://doi.org/10.1016/j.neulet.2014.12.050

[3] R. Mullur, Y.Y. Liu and G.A. Brent, Thyroid hormone regulation of metabolism, Physiological Reviews 94 (2014), 355-382. https://doi.org/10.1152/physrev.00030.2013

[4] F.S. Greenspan and B.J. Dong, Thyroid and Antithyroid Drugs, in: Basic Clinical Pharmacology, 2nd ed., Ed.: B.G. Katzung, Los Altos, California: Lange, 1984.

[5] A.W. White, P. Handler and E.L. Smith, Principles of Biochemistry, 4th ed., New York: McGraw-Hill, 1968.

[6] R.S. Vardanyan and V.J. Hruby, Thyroid Hormone and Antithyroid Drugs, in: Synthesis of Essential Drugs, Eds.: R.S. Vardanyan, V.J. Hruby, Amsterdam: Elsevier, 2006, pp. 337-342 . https://doi.org/10.1016/B978-044452166-8/50025-X

[7] L. Jurd, The iodination of tyrosine and its derivatives, J. Am. Chem. Soc. 77(21) (1955), 5747. https://doi.org/10.1021/ja01626a091

[8] R.L. Perlman and H. Edelhoch, The formation of diiodotyrosine in iodinated human serum albumin, The Journal of Biological Chemistry 242(10) (1967), 2416-2422. https://doi.org/10.1016/S0021-9258(18)95977-6 
[9] S. Yanagida, A. Kaname and N. Murakami, Quantum chemistry-based verification of antioxidative action of iodide in mitochondria, Integrative Molecular Medicine 6 (2019) 1-6. https://doi.org/10.15761/IMM.1000388

[10] J.J.P. Stewart, Optimization of parameters for semi empirical methods I, J. Comput. Chem. 10 (1989), 209-220. https://doi.org/10.1002/jcc.540100208

[11] J.J.P. Stewart, Optimization of parameters for semi empirical methods II, J. Comput. Chem. 10 (1989), 221-264. https://doi.org/10.1002/jcc.540100209

[12] A.R. Leach, Molecular Modeling, Essex: Longman, 1997.

[13] W. Kohn and L.J. Sham, Self-consistent equations including exchange and correlation effects, Phys. Rev. 140 (1965), 1133-1138. https://doi.org/10.1103/PhysRev.140.A1133

[14] R.G. Parr and W. Yang, Density Functional Theory of Atoms and Molecules, London: Oxford University Press, 1989.

[15] A.D. Becke, Density-functional exchange-energy approximation with correct asymptotic behavior, Phys. Rev. A 38 (1988), 3098-3100. https://doi.org/10.1103/PhysRevA.38.3098

[16] S.H. Vosko, L. Vilk and M. Nusair, Accurate spin-dependent electron liquid correlation energies for local spin density calculations: a critical analysis, Can. J. Phys. 58 (1980), 1200-1211. https://doi.org/10.1139/p80-159

[17] C. Lee, W. Yang and R.G. Parr, Development of the Colle-Salvetti correlation-energy formula into a functional of the electron density, Phys. Rev. B 37 (1988), 785-789. https://doi.org/10.1103/PhysRevB.37.785

[18] SPARTAN 06, Wavefunction Inc., Irvine CA, USA, 2006.

[19] L.N. Ferguson, The Modern Structural Theory of Organic Chemistry, New Delhi: Prentise-Hall, 1969.

[20] I. Fleming, Frontier Orbitals and Organic Chemical Reactions, London: Wiley, 1976. 